International Conference on the Physics of Multiply

Charqed Ions / International Workshop on ECR Ion Sources Sept. 14-16, 1988, Grenoble, France

$$
\text { CONF-880955-3 }
$$

\title{
ELECTRON CAPTURE BY MULTICHARGED IONS AT EV ENERGIES
}

C. C. Havener, M. S. Huq, F. W. Meyer, and R. A. Phaneuf

Gak Ridge National Laboratory, Oak Ridge, Tennessee 37831-6372 USA

Résumé

CONF-880955--3

DISCLAIMER

DE89 001156

\begin{abstract}
This report was prepared as an account of work sponsored by an agency of the United States Government. Neither the United States Government nor any agency thereof, nor any of their employees, makes any warranty, express or implied, or assumes any legal liability or responsibility for the accuracy, completeness, or usefulness of any information, apparatus, product, or process disclosed, or represents that its use would not infringe privately owned rights. Reference herein to any specific commercial product, process, or scrvice by trade name, trademark, manufacturer, or otherwise does not necessarily constitute or imply its endorsement, recommendation, or favoring by the United States Government or any agency thereof. The views and opinions of authors expressed herein do not necessarily state or reflect those of the United States Government or any agency thereof.
\end{abstract}

Abstract - A multicharged ion-atom merged-beams apparatus has been used in conjunction with the ORNL-ECR ion source to measure accurate absolute electron-capture cross sections in the energy range from below $1 \mathrm{eV} / \mathrm{amu}$ to $1500 \mathrm{eV} / \mathrm{amu}$. Measurements for $\mathrm{N}^{3+, 4+, 5+}+\mathrm{H}(\mathrm{D})$ collisions indicate good agreement with avallable theoretical calculations. However, measurements with $0^{5+}+\mathrm{H}(\mathrm{D})$ shnw an unexpected low-energy behavior which may be attributable to the ion-induced-dipole attraction between the reactants. scaled Landau-Zener calculations presented here identify a transfer plus excitation channel which has the correct energy dependence at low energies. This finding suggests the need for a comprehensive coupled channel calculation which would include such product states.

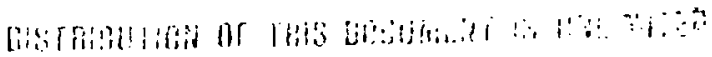

$1-$ INTRODUCTION

MASTEA th

The inelastic process whereby a multicharged ion captures an electron from a neutral atom is important in environments containing both highly charged ions and neutrals. Cross sections for this electron-capture process at low energies are typically large $\left(>10^{-15} \mathrm{~cm}^{2}\right)$ and selectively populate excited states of the multiply charged ion. Such collisions, therefore, are important for plasmas of thermonuclear fusion, astrophysics, multicharged ion sources, and the development of $X$-ray lasers.

Only very limited data are available for electron capture by multicharged fons colliding with neutrals in the velocity range (v<0.1 a.u.). In such low-velocity collisions, the relative nuclear motion between collision partners is slow compared to the orbital motion of the active electrons of the system. Electrons of the temporary quasi-molecule formed in the collision have sufficient time to adjust: to the changing interatomic field as the nuclei 


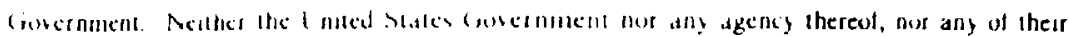
employees, makes any warranty, express or implied, or assumes any legal liability or responsi. bility for the accuracy, completeness, or usefulness of any information, apparatus, product, or process disclosed, or represents that its use would not infringe privately owned rights. Reference herein to any spocific commercial product, process, or service by trade name, trademark, manufacturer, or otherwise does nut necessarily constitute or imply its endorsement, recommendation. or favoring by the United States Government or any agency thereof The views and opinions of authors expressed herein do not necessarily state or reflect those of the United States Government or any agency thereof.

Abstract - A multicharged ion-atom merged-beams apparatus has been used in conjunction with the ORNL-ECR ion source to measure accurate absolute electron-capture cross sections in the energy range from below 1 eV/amu to $1500 \mathrm{eV} / \mathrm{amu}$. Measurements for $\mathrm{N}^{3+.4+, 5+}+\mathrm{H}(\mathrm{O})$ collisions indicate good agreement with avallable theoretical calculations. However, measurements with $0^{5+}+H(D)$ show an unexpected low-energy behavior which may be attributable to the ion-induced-dipole attraction between the reactants. Scaled Landau-Zener calculations presented here identify a transfer plus excitation channel which has the correct energy dependence at low energies. This finding suggests the need for a comprehensive coupled channel calculation which would include such product states.

\section{1 - INTROOUCTION \\ I - INTROOUCTION}
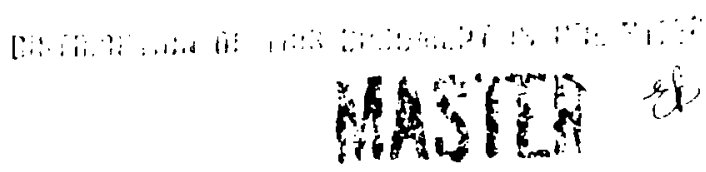

The inelastic process whereby a multicharged ion captures an electron from a neutral atom is important in environments containing both highly charged lons and neutrals. Cross sections for this electron-capture process at low energies are typically large $\left(>10^{-15} \mathrm{~cm}^{2}\right)$ and selectively populate excited states of the multiply charged ion. Such collisions, therefore, are important for plasmas of thermonuclear fusion, astrophysics, multicharged ion sources, and the development of $X$-ray lasers.

Only very limited data are available for electron capture by multicharged fons colliding with neutrals in the velocity range (v<0.1 a.u.). In such low-velocity collisions, the relative nuclear motion between collision partners is slow compared to the orbital motion of the active electrons of the system. Electrons of the temporary quasi-molecule formed in the collision have sufficient time to adjust to the changing interatomic field as the nuclei approach and separate. Coupled stationary-state calculations 11,21 , while successful at higher energies, do not necessarily give consistent predictions at lower energies. In such calculations, the internuclear motion has generally been treated classically. It would seem that fuil quantal calculations $13-5 /$ may be required for accurate predictions at the lowest energies, but such methods have remained until recently $/ 6,7 /$ essentially untested. At these low energies there has been speculation $/ 3,8 /$ that the ion-induced dipole attraction between the reactants may cause orbiting trajectories $19 \%$. Cross sections due to this process are predicted classically to have a $1 / v$ dependence at lower energies.

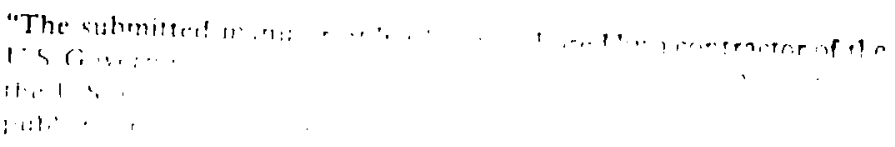


Most of the exfa-imental work on such collision processes has been based on ion-beam gas-target methods, which have generally been limited to energies above $100 \mathrm{eV} / \mathrm{amu}$. Some experiments at lower energies have been performed by Phaneuf et al. /10/ using a pulsedlaser-produced plasma as a source of slow multiply charged lons. In the 10 to $100 \mathrm{eV} / \mathrm{amu}$ energy regime they found that for few-electron systems there exists no simple scaling of the cross sections with charge or velocity, so that each system must be investigated separately. The strong influence of the ionic core has been seen in theoretical calculations $/ 11,12 /$ and measurements /13/ of substate distributions at energies less than a few $\mathrm{keV} / \mathrm{amu}$.

To address the need for experimental measurements in this energy range, an ion-atom mergedbeam apparatus has been developed $16 /$ at Oak Ridge National Laboratory to measure absolute total electron capture cross sections for multicharged ions colliding with atomic hydrogen in the energy range of 1 to $1000 \mathrm{ev} / \mathrm{amu}$.

\section{2 - EXPERIMENTAL}

The merged-beam method is well suited for such measurements in this energy range. Details are presented in ref. 6 . In this technique, beams of neutral atoms and multicharged ions each having laboratory energies in the keV range are merged, resulting in a relative velocity of the two beams that can be "tuned" over a large range. The relative energy in the center-ofmass frame is given by

$$
E_{\text {rel }}(\mathrm{eV} / \mathrm{amu})=\frac{E_{1}}{m_{1}}+\frac{E_{2}}{m_{2}}-2\left(\frac{E_{1}}{m_{1}} \frac{E_{2}}{m_{2}}\right)^{1 / 2} \cos \theta
$$

where $E_{j}$ and $m_{j}$ denote the energy and mass of each beam, and $\theta$ is their angle of intersection. For perfectly collinear beams, $\theta=0$ and $E_{r e l}$ can be made zero for $E_{1} / m_{1}=E_{2} / m_{2}$.

Figure 1 is a simplified schematic of the apparatus. The multicharged ion beam $x a+$ is merged electrostatically with a neutral $H$ or $D$ beam. The merged beams interact in a fieldfree region for a distance of $80.8 \mathrm{~cm}$, after which the primary beams are magnetically separated from each other and from the product or "signal" $H(D)^{+}$ions. The $X(q-1)+$ product of the reaction is not measured separately, but is collected together with the primary $x a+$ in a large faraday cup. The neutral beam intensity is measured by secondary-electron emission from a stainless steel plate, and the signal $\mathrm{H}^{+}$or $\mathrm{D}^{+}$ions are recorded by a channel electron multiplier (CEM) operated in pulse-counting mode. A pure ground-state beam of $H$ or $D$ atoms is produced by passing a $6-9 \mathrm{keV}$ beam of $\mathrm{H}^{-}$or $\mathrm{D}^{-}$ions through the optical cavity of a 1.05- $\mathrm{m} \mathrm{md}$ :YAG laser, where up to $600 \mathrm{~W}$ of continuous power circulates and typically $0.5 \%$ of the negative ions undergo photodetachment. A nearly parallel beam of $H(D)$ atoms is produced in the merge-path with a diameter of 2-4 mm FWHM and an intensity of 10-20 (particle) na. The divergence of this beam is typically less than $0.2^{\circ}$. A 50-90 keV, 2-5 $\mu A$ beam of $X 9+$ ions is produced by the ORNL-ECR with a typically 6-8 m FWHM in the merge path and a divergence of less than $0.5^{\circ}$.

Figure 2 shows the relative energy in the center-of-mass frame obtained for three different neutral-beam conditions for collisions involving $0^{5+}$. To change the energy, Erel, the energy of the $0^{5+}$ is varied while the velocity of the $H$ or $D$ beam is held constant. It is clear from the figure that a wide range of relative energies is obtainable with this apparatus. As is shown in the figure, an interaction energy of $100 \mathrm{eV} / \mathrm{amu}$ may be obtained several different ways: $D$ faster, $D$ slower, and $H$ faster than the $0^{5+}$ beam.

These different laboratory cond1- 
The merged-beam method is well suited for such measurements in this energy range. Details are presented in ref. 6. In this technique, beams of neutral atoms and multicharged ions each having laboratory energies in the keV range are merged, resulting in a relative velocity of the two beams that can be "tuned" over a large range. The relative energy in the center-ofmass frame is given by

$$
E_{\text {rel }}(\mathrm{eV} / \mathrm{amu})=\frac{E_{1}}{m_{1}}+\frac{E_{2}}{m_{2}}-2\left(\frac{E_{1}}{m_{1}} \frac{E_{2}}{m_{2}}\right)^{1 / 2} \cos \theta
$$

where $E_{f}$ and $m_{f}$ denote the energy and mass of each beam, and $\theta$ is their angle of intersection. For perfectly collinear beams, $\theta=0$ and $E_{\text {rel }}$ can be made zero for $E_{1} / m_{1}=E_{2} / m_{2}$.

Figure 1 is a simplified schematic of the apparatus. The multicharged ton beam $x a+$ is merged electrostatically with a neutral $H$ or $D$ beam. The merged beams interact in a fieldfree region for a distance of $80.8 \mathrm{~cm}$, after which the primary beams are magnetically separated from each other and from the product or "signal" $H(D)^{+}$ions. The $X(q-1)+$ product of the reaction is not measured separately, but is collected together with the primary $x a+$ in a large faraday cup. The neutral beam intensity is measured by secondary-electron emission from a stainless steel plate, and the signal $\mathrm{H}^{+}$or $\mathrm{D}^{+}$ions are recorded by a channel electron multiplier (CEM) operated in pulse-counting mode. A pure ground-state beam of $H$ or $D$ atoms is produced by passing a 6-9 keV beam of $\mathrm{H}^{-}$or $\mathrm{D}^{-}$ions through the optical cavity of a 1.06- $\mu \mathrm{m}$ Nd:YAG laser, where up to $600 \mathrm{~W}$ of continuous power circulates and typically $0.5 \%$ of the negative ions undergo photodetachment. A nearly parallel beam of $H(D)$ atoms is produced in the merge-path with a diameter of 2-4 mm FWHM and an intensity of 10-20 (particle) na. The divergence of this beam is typically less than $0.2^{\circ}$. A 50-90 keV, 2-5 $\mu \mathrm{A}$ beam of $\mathrm{Xq+}$ ions is produced by the ORNL-ECR with a typically 6-8 $\mathrm{mm}$ FWHM in the merge path and a divergence of less than $0.5^{\circ}$.

Figure 2 shows the relative energy in the center-of-mass frame obtained for three different neutral-beam conditions for collistons involving $0^{5+}$. To change the energy, Erel, the energy of the $0^{5+}$ is varied while the velocity of the $H$ or $D$ beam is held constant. It is clear from the figure that a wide range of relative energies is obtainable with this apparatus. As is shown in the figure, an interaction energy of $100 \mathrm{eV} / \mathrm{amu}$ may be obtained several different ways: D faster, $D$ slower, and $H$ faster than the $0^{5+}$ beam. These different laboratory conditions provide important diagnostic checks on the measurements. 
ORNL-DWG 88-11570

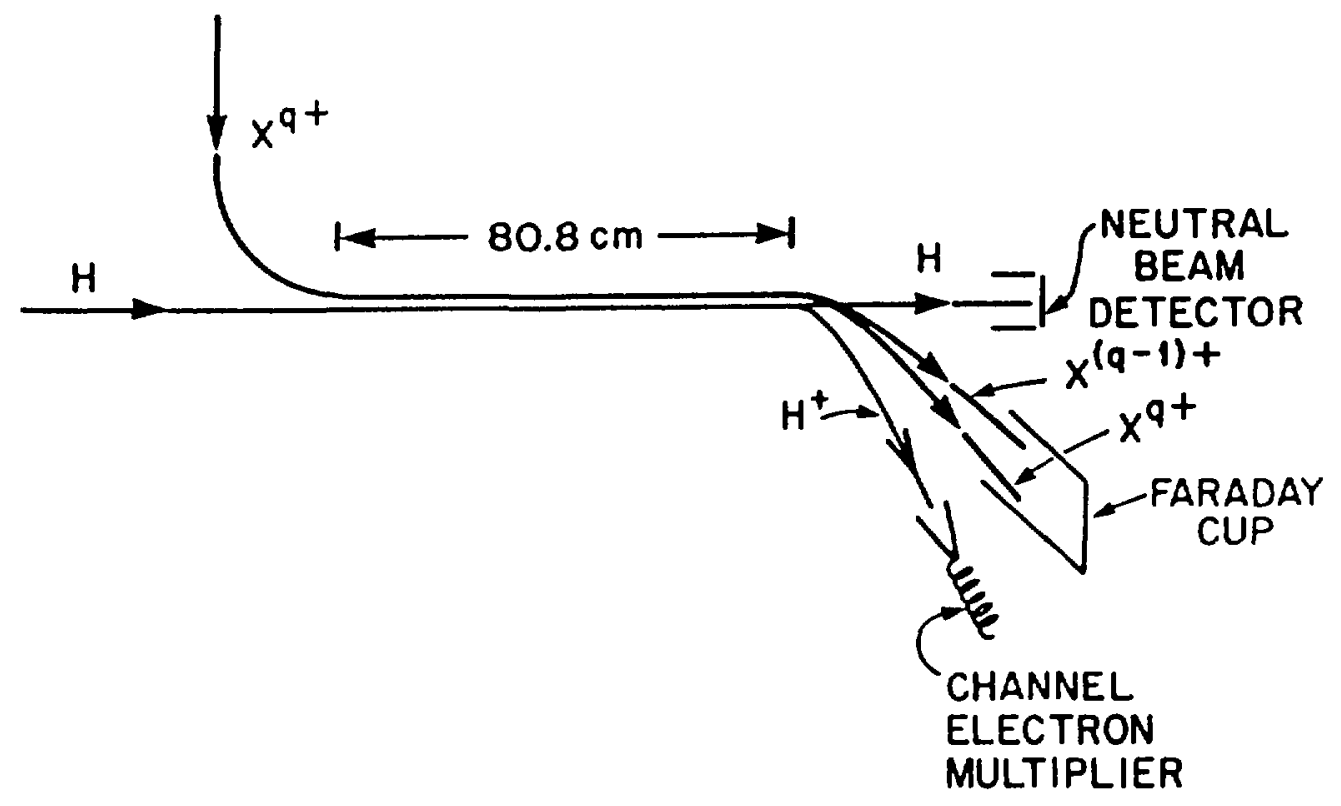

Fig. 1. Simplifled schematic of the ion-neutral merged-beam apparatus.

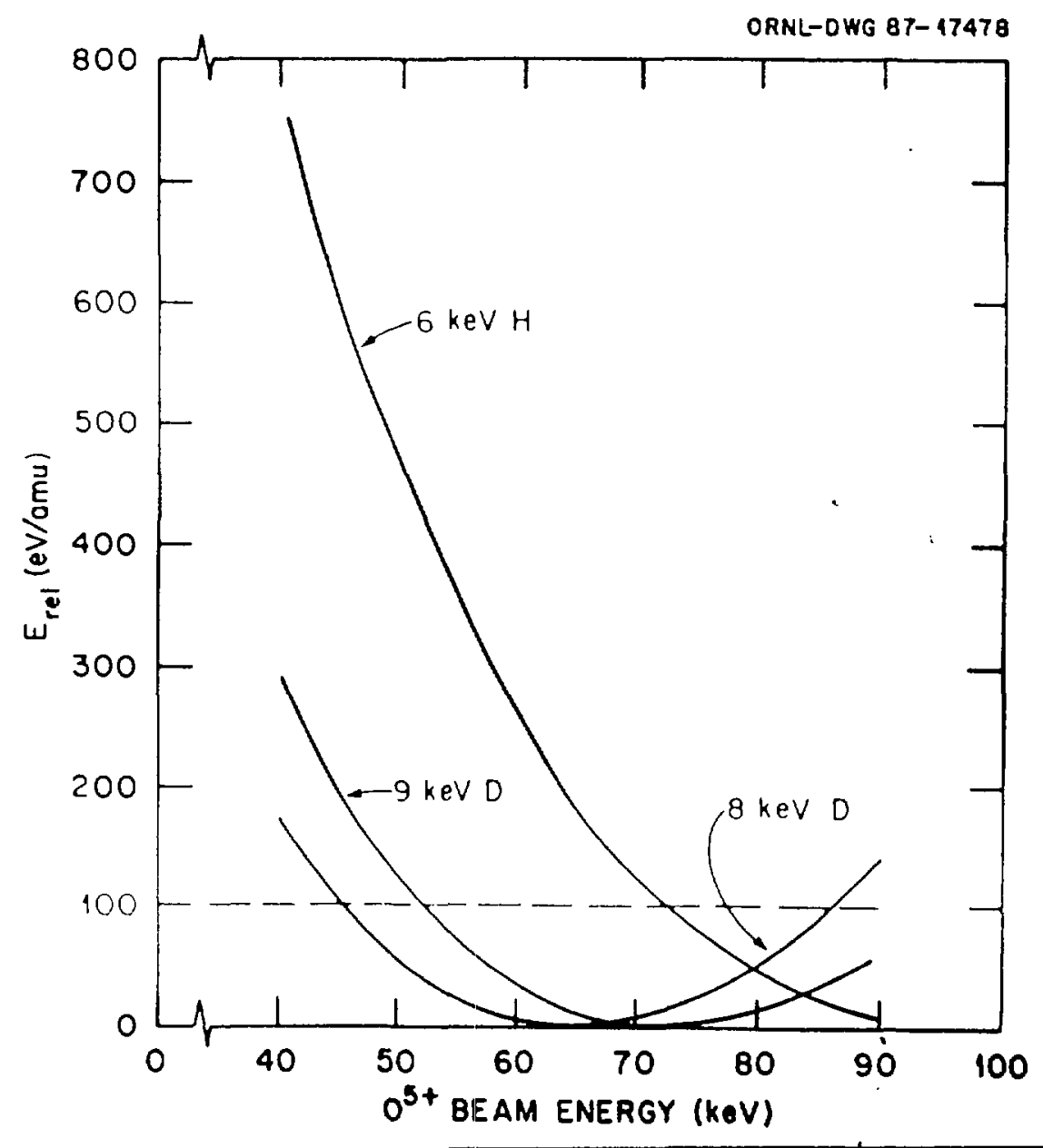




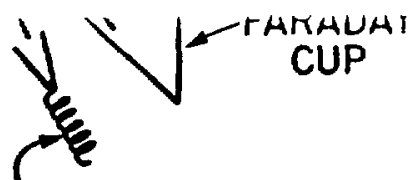

\section{CHANNEL ELECTRON MULTIPLIER}

Fig. 1. Simplified schematic of the ion-neutral merged-beam apparatus.

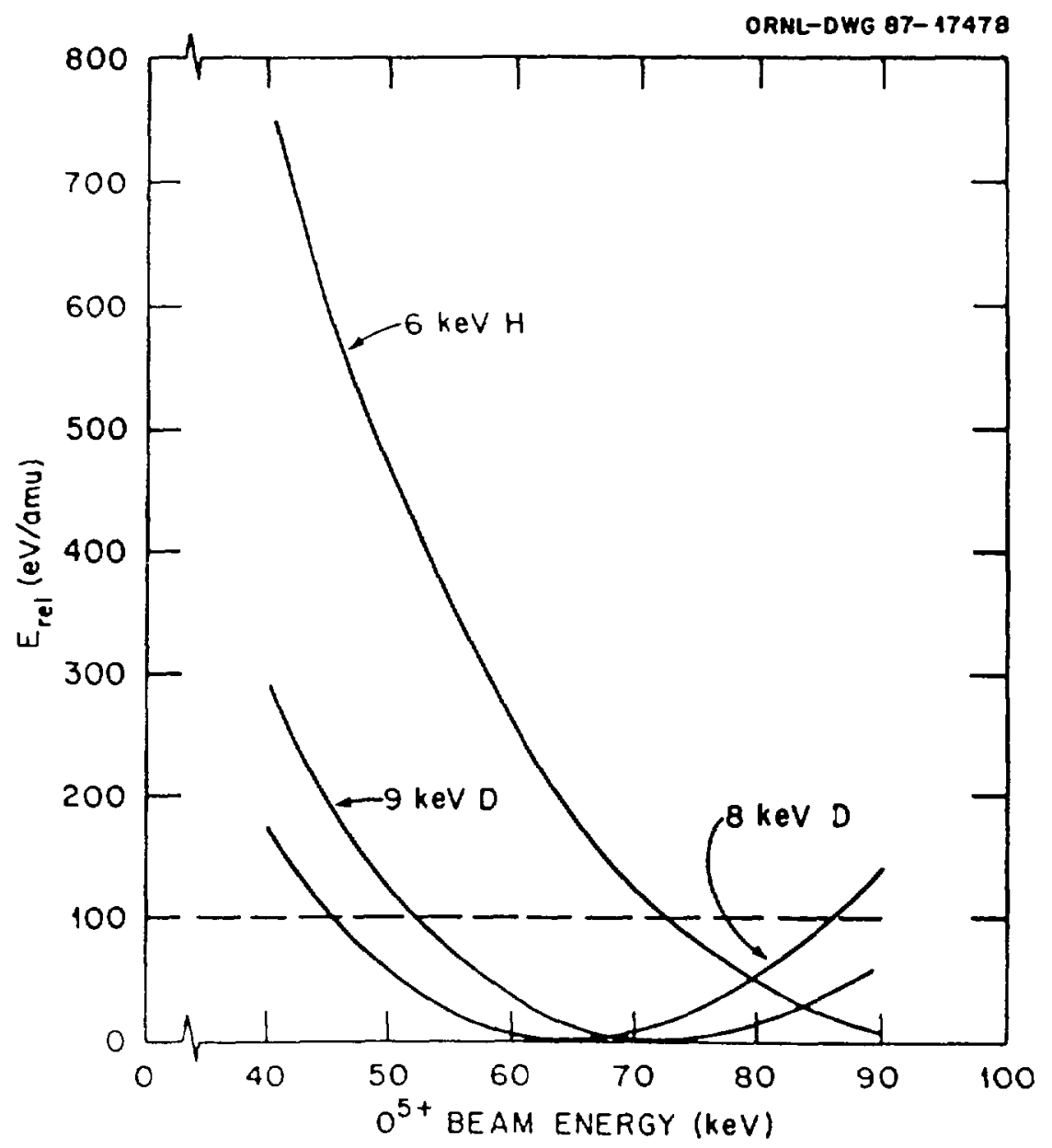

Fig. 2. The relative energy, Erel, obtainable in the present apparatus for $6 \mathrm{keV} \mathrm{H,} 8 \mathrm{keV}$, and $9 \mathrm{keV} D$ neutral beams as function of the $0^{5+}$ beam energy. The intersections of the horizontal line at $100 \mathrm{eV} / \mathrm{amu}$ with the different $H(D)$ curves lllustrate that several different laboratory conditions can be used to measure the cross section at aiven Erel. 
An important advantage of the merged-beam technique is the large deamplification of the interaction-energy uncertainty resulting from the energy uncertainties in the primary beams. In Fig. 2, the slope of the curves is just $\Delta E_{r e l} / \Delta E_{2}$, where $\Delta E_{2}$ is the energy uncertainty in the $0^{5+}$ beam. As $E_{\text {rel }}$ decreases, the slope decreases, reducting the uncertainty in $E_{\text {rel }}$. For an estimated uncertainty of $10 \mathrm{eV}$ in the neutral beam energy and $100 \mathrm{eV}$ in the $0^{5+}$ beam energy, the estimated uncertainty due to each beam is less than $1 \mathrm{eV} / \mathrm{amu}$ at $E_{\mathrm{rel}}=100 \mathrm{eV} / \mathrm{amu}$ and $0.1 \mathrm{eV} / \mathrm{amu}$ at $E_{\mathrm{rel}}=1.0 \mathrm{eV} / \mathrm{amu}$.

Another advantage of the merged-beam technique in low-energy measurements is the potentially large angular collection of the reaction products. The low energy electron capture collisions under study are exoergic, and, since both products are positively charged, they can undergo significant angular scattering in the center-of-mass frame $/ 14 /$. In this respect the merged-beam technique is ideal since the angular scattering in the center-of-mass frame becomes significantly compressed in the laboratory frame, in which the product lons are collected. The apparatus was designed to have as large an angular collection as possible. An average angular collection in the laboratory frame along the $80.8 \mathrm{~cm}$ merge path was determined by detailed trajectory calculations $/ 6 /$ to be $1.8^{\circ}$. The resultant angular collection in the center-of-mass frame is shown in Fig. 3. Note that for measurements performed with a $9 \mathrm{keV} D$ beam at an $E_{\text {rel }}=1 \mathrm{eV} / \mathrm{amu}$ product trajectories with up to $90^{\circ}$ scattering in the center-of-mass frame are collected.

Electron-capture cross sections are determined by measuring the rate of $\mathrm{H}^{+}\left(\mathrm{D}^{+}\right)$product fons produced by the beam-beam interaction over the merged length L. The cross-section value is calcuiated at each velocity from the directly measurable quantities through the equation

$$
\sigma=\frac{R Y q e^{2}}{I_{1} I_{2}} \frac{v_{1} v_{2}}{v_{r}} \frac{1}{L\langle F\rangle}
$$

ORNL-DWG $87-17477$

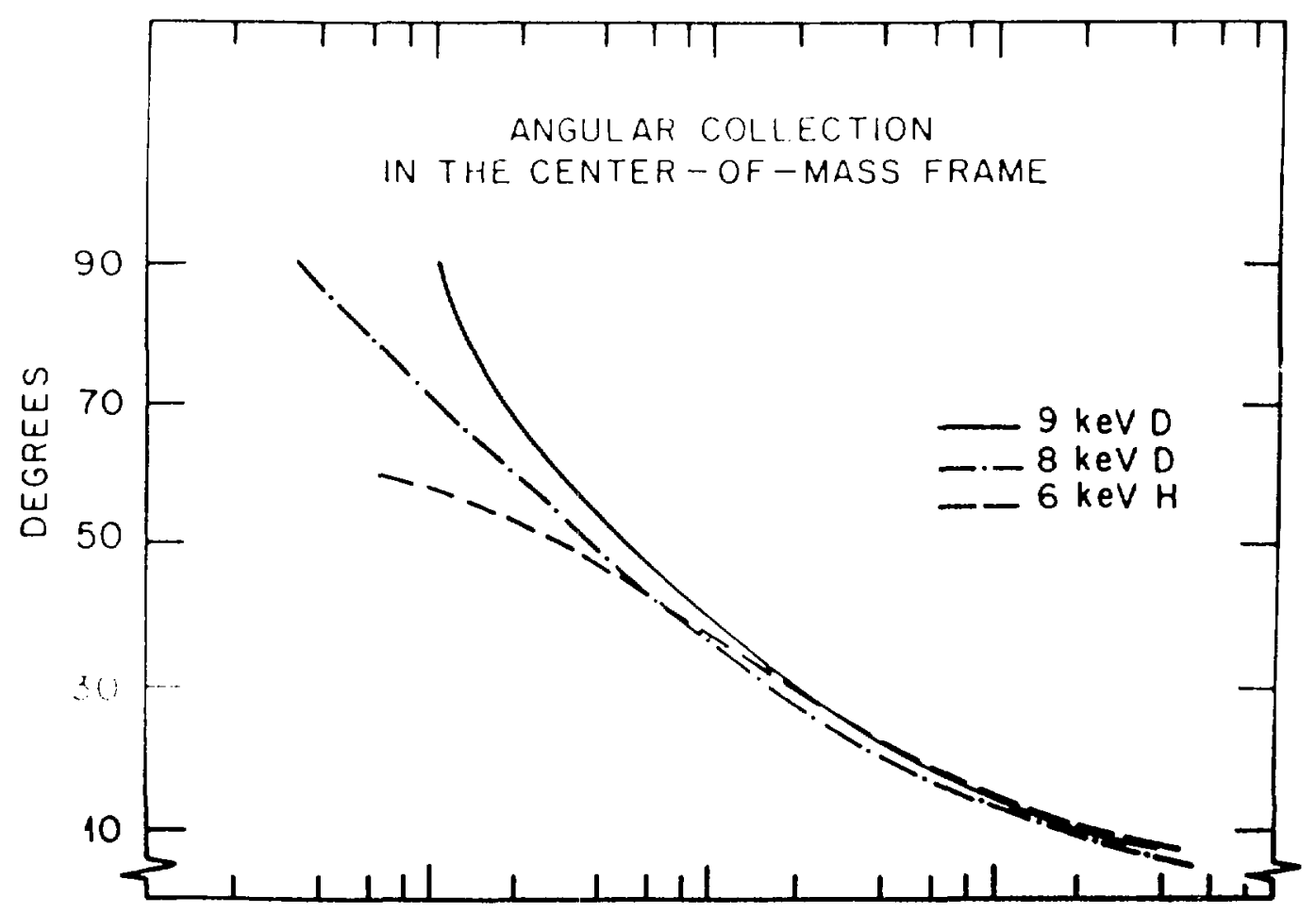


becomes significantly compressed in the laboratory frame, in which ine product ions are collected. The apparatus was designed to have as large an angular collection as possible. An average angular collection in the laboratory frame along the $80.8 \mathrm{~cm}$ merge path was determined by detailed trajectory calculations $/ 6 /$ to be $1.8^{\circ}$. The resultani angular collection in the center-of-mass frame is shown in Fig. 3. Note that for measurements performed with a $9 \mathrm{keV} D$ beam at an $E_{r e l}=1 \mathrm{eV} / \mathrm{amu}$ product trajectories with up to $90^{\circ}$ scattering in the center-of-mass frame are collected.

Electron-capture cross sections are determined by measuring the rate of $\mathrm{H}^{+}\left(\mathrm{D}^{+}\right)$product lons produced by the beam-beam interaction over the merged length $L$. The cross-section value is calculated at each velocity from the directly measurable quantities through the equation

$$
0=\frac{R \gamma q e^{2}}{I_{1} I_{2}} \frac{v_{1} v_{2}}{v_{r}} \frac{1}{L\langle F\rangle}
$$

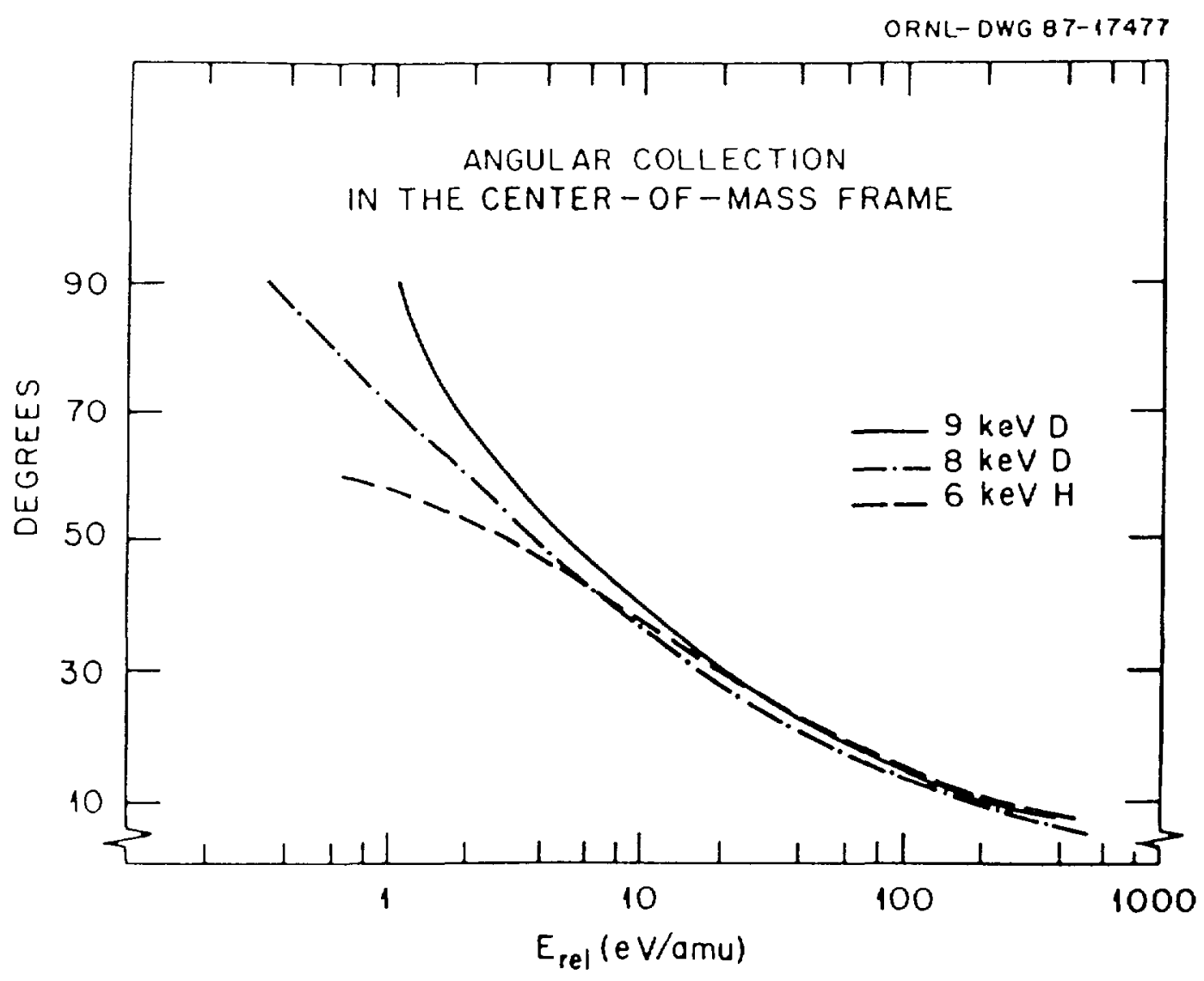

Fig. 3. The estimated angular collection of the reaction products in the center-of-mass frame as a function of $E_{r e l}$ for measurements performed with a $6 \mathrm{keV} \mathrm{H}, 8 \mathrm{keV} D$, and a $9 \mathrm{keV} D$ neutral beam. 
where $R, I_{1}$, and $I_{2}$ correspond to the measured count rate, effective current produced by neutral particles, and $0^{5+}$ current, respectively. The true neutral "current" is given by $I_{1} / Y, Y$ being the measured effective secondary-electron emission coefficient for the neutralparticle detector. The quantity $q$ is the charge state of the inciderit ion, $a$ is the electronic charge, and $v_{r}$ is the relattve velocity of the two beams, traveling at yelocities $v_{1}$ and $v_{2}$. $\langle F\rangle$ is the effective form factor $/ 6 /$ and is a measure of the spatial overlap of the two beams along the merged path $L$.

To obtain a value for $\langle F\rangle$, the beam-beam overlap is measured in two dimensions at four different locations along the merged path. Measurements were performed by two mechanical knifeedge scanners and two rotating-wire monitors, each performing horizontal and vertical scans (see ref. 6). The secondary emission coefficient for the neutral detector was calibrated (a process taking several hours) in situ and found to have a slight dependence on neutral beam velocity and vacuum history. For typical operating conditions at an interaction energy of $25 \mathrm{eV} / \mathrm{amu}$ for $\mathrm{C}^{5+}+\mathrm{D}$, the signal rate, $R$, is typically $100 \mathrm{~Hz}$ and must be detected in the presence of a background of $6 \mathrm{kHz}$. This background is due to the neutral beam stripping on background gas. Therefore, vacuum in the mcrge section had to be maintained as low as possible, typically 1-2 × 10-10 Torr with beams. To separate the signal from the background, a two-beam modulation scheme $/ 6 /$ was used. Since the signal rate produced by the beam-beam interaction is proportional to the relative velocity of the beams (for a constant cross section), the signal-to-background ratio determined the lowest energies at which measurements could be made. If the cross section, in fact, al so decreases with decreasing energy, measurements become more difficult at lower energies. The lower-energy limit imposed by the apparatus itself is approximately $0.2 \mathrm{eV} / \mathrm{amu}$, and is a consequence of the finite angular divergence of the interacting beams.

\section{3 - CROSS-SECTION MEASUREMENTS}

The merged-beam apparatus has been used $/ 6,7 /$ to measure total electron capture cross sections for $\mathrm{O}^{5+}$ and $\mathrm{N}^{3+, 4+, 5+}$ colliding with ground state $\mathrm{H}(\mathrm{D})$ in the energy range $<1.0 \mathrm{eV} / \mathrm{amu}$ to $\approx 1500 \mathrm{eV} / \mathrm{amu}$. The measurements for $\mathrm{O}^{5+}$ and $\mathrm{N}^{5+}$ are compares to other measurements and theory in Figs. 4 and 5 , respectively. The error bars shown denote the relative uncertainty estimated at a $90 \%$ confidence level. The total absolute uncertainty is indicated by extended error bars on a few points in Fig. 4. For all systems studied the measurements were performed at sufficiently high $E_{\text {rel }}$ to overlap with existing experimental measurements. Agreement with previous measurements is shown to be good, especially in the $0^{5+}$ system (see Fig. 4) where low-energy measurements by Phaneuf et al. $/ 10 /$ extend down to $80 \mathrm{eV} / \mathrm{amu}$, and, thereby, provide an extended range of comparison. These measurements were performed with a laser-ton source and a hydrogen oven as target. In this energy range there are also two unpublished data points by. Meyer et al., measured with the ORNL-ECR ion source and the same hydrogen oven (see ref. 15). The $0^{5+}$ and the $N^{4+}$ lons are L1-IIke and, therefore, free of metastables, allowing measurements with these ions to be directly compared with theoretical calculations where they exist.

For the $0^{5+}$ system there is good agreement with the unpublished full quantal theoretical calculations of Bottcher and Heil (see ref. 3) which include capture to the $n=4$ molecular $\Sigma$ and $\Pi$ states for the $\mathrm{O}^{4+}+\mathrm{H}^{+}$system. However, the measured cross section unexpectediy decreased when the measurements were extended below $10 \mathrm{eV} / \mathrm{amu}$. This decrease is in contradiction to the energy behavior shown in the full quantal calculations of Gargaud and At first this cherud 
presence of a background of $6 \mathrm{kHz}$. This background is due to the neutral beam stripping on background gas. Therefore, vacuum in the merge section had to be maintained as low as possible, typically 1-2 × 10-10 Torr with beanij. To separate the signal from the background, a two-beam modulation scheme $/ 6 /$ was used. Since the signal rate produced by the beam-beam interaction is proportional to the relative velocity of the beams (for a constant cross section), the signal-to-background ratio determined the iowest energies at which measurements could be made. If the cross section, in fact, also decreases with decreasing energy, measurements become more difficult at lower energies. The lower-energy limit imposed by the apparatus itself is approximately $0.2 \mathrm{ev} / \mathrm{amu}$, and is a consequence of the finite angular divergence of the interacting beams.

\section{3 - CROSS-SECTION MEASUREMENTS}

The merged-beam apparatus has been used /6,7/ to measure total electron capture cross sections for $0^{5+}$ and $\mathrm{N}^{3+, 4+, 5+}$ colliding with ground state $H(D)$ in the energy range $<1.0 \mathrm{eV} / \mathrm{amu}$ to $\approx 1500 \mathrm{eV} / \mathrm{amu}$. The measurements for $0^{5+}$ and $\mathrm{N}^{5+}$ are compared to other measurements and theory in Figs. 4 and 5 , respectively. The error bars shown denote the relative uncertainty estimated at a $90 \%$ confidence level. The total absolute uncertainty is indicated by extended error bars on a few points in Fig. 4. For all systems studied the measurements were performed at sufficiently high $E_{r e l}$ to overlap with existing experimental measurements. Agreement with previous measurements is shown to be good, especially in the $0^{5+}$ system (see Fig. 4) where low-energy measurements by Phaneuf et al. $/ 10 /$ extend down to $80 \mathrm{eV} / \mathrm{amu}$ and, thereby, provide an extended range of comparison. These measurements were performed with a laser-ion source and a hydrogen oven as target. In this energy range there are also two unpublished data points by Meyer et al., measured with the ORNL-ECR ion source and the same hydrogen oven (see ref. 15). The $0^{5+}$ and the $\mathrm{N}^{4+}$ ions are L1-like and, therefore, free of metastables, allowing measurements with these ions to be directly compared with theoretical calculations where they exist.

For the $0^{5+}$ system there is good agreement with the unpublished full quantal theoretical calculations of Bottcher and Heil (see ref. 3) which include capture to the n=4 molecular $\Sigma$ and $\Pi$ states for the $\mathrm{O}^{4+}+\mathrm{H}^{+}$system. However, the measured cross section unexpectedly decreased when the measurements were extended below $10 \mathrm{eV} / \mathrm{amu}$. This decrease is in contradiction to the energy behavior shown in the full quantal calculations of Gargaud and McCarroll /16/ which predict an increase in $\sigma_{54}$ as Erel decreases. At first this observed decrease was thought to be due io a loss of collected signal as a result of large angular scattering of the products. The angular collection of the apparatus in this energy range is less than $20^{\circ}$ (see Fig. 3). However, data taken under differing laboratory conditions with significantly different angular collection in the center-of-mass frame (see Fig. 6) show the same energy dependence, leading us to conclude that the angular collection of products is sufficient. 


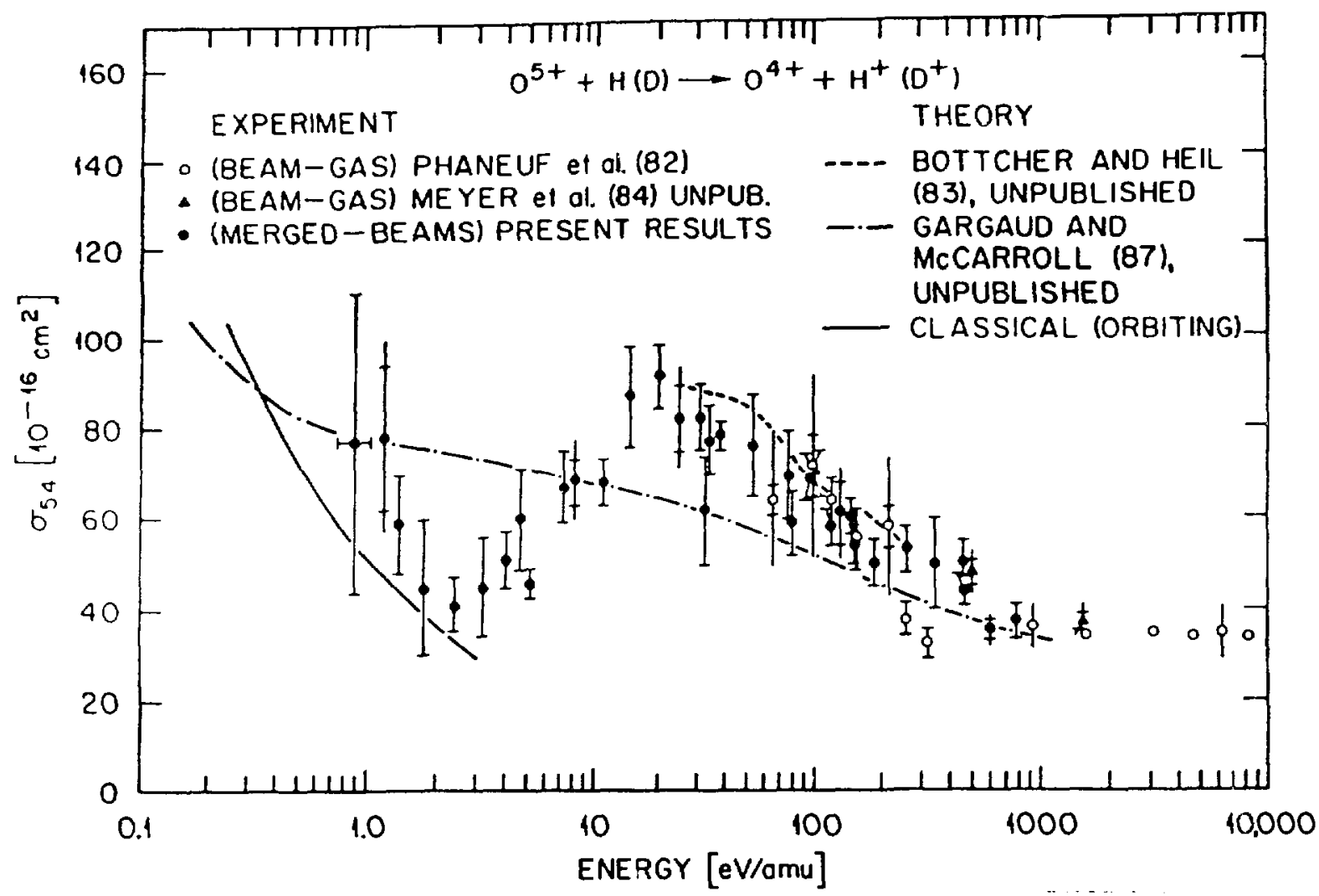

Fig. 4. Comparison of merged-beams data for electron capture in $0^{5+}+H$ collisions with previous measurements and theory. Select data are presented with inner and outer error bars denoting relative and estimated total experimental uncertainties estimated at a $90 \%$ confidence level. The energy uncertainty is denoted by a horizontal bar at the lowest energy, where the uncertainty is relatively the largest.

ORNL-OWG $88-8580$

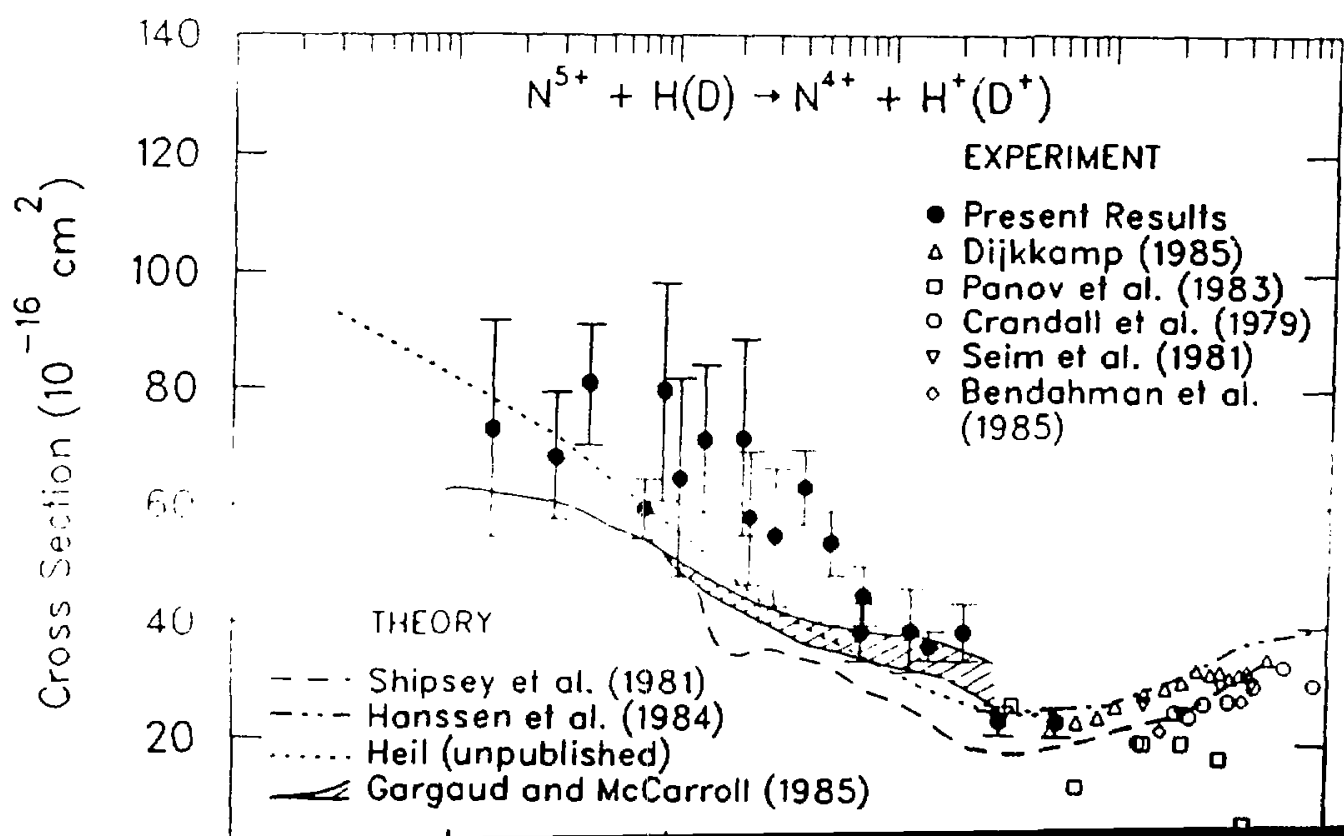




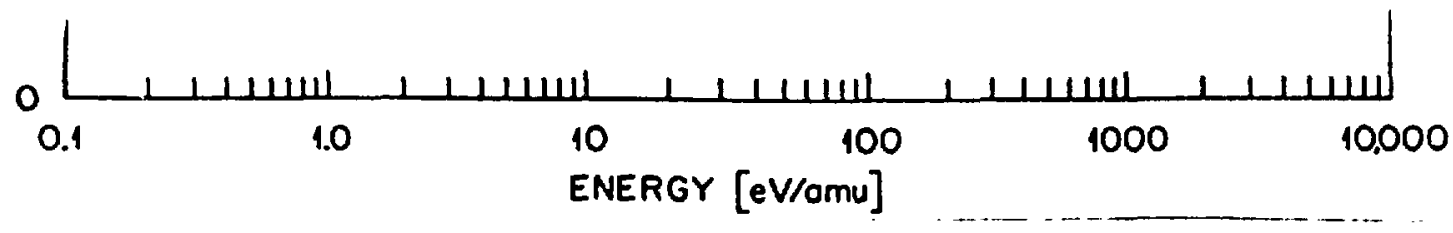

Fig. 4. Comparison of merged-beams data for electron capture in $0^{5+}+\mathrm{H}$ collisions with previous measurements and theory. Select data are presented with inner and outer error bars denoting relative and estimated total experimental uncertainties estimated at a $90 \%$ confidence level. The energy uncertainty is denoted by a horizontal bar at the lowest energy, where the uncertainty is relatively the largest.

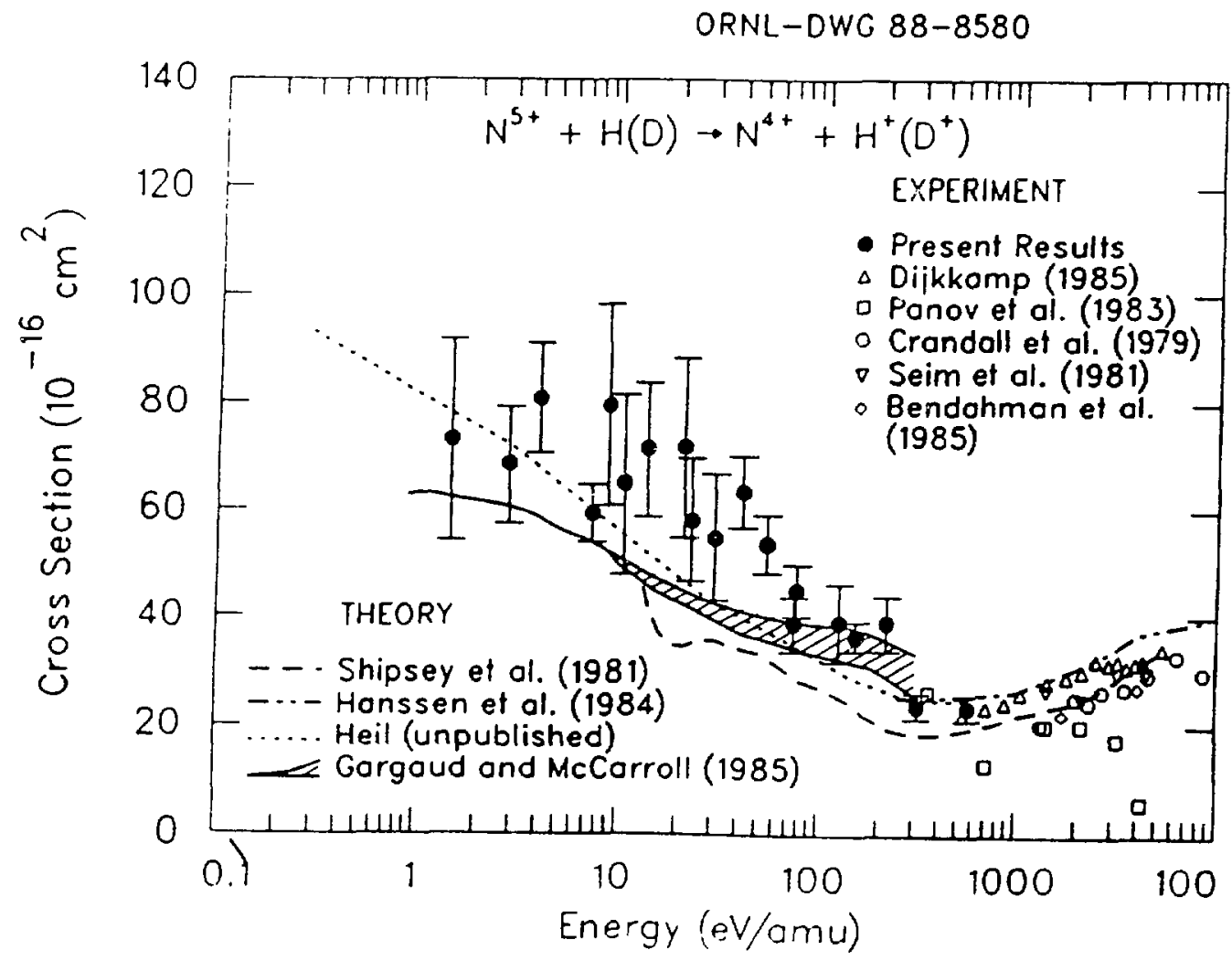

Fig. 5. Comparison of merged beams data for electron capture in $\mathrm{N}^{5}+\mathrm{H}$ collistons with previous measurements and theory. 


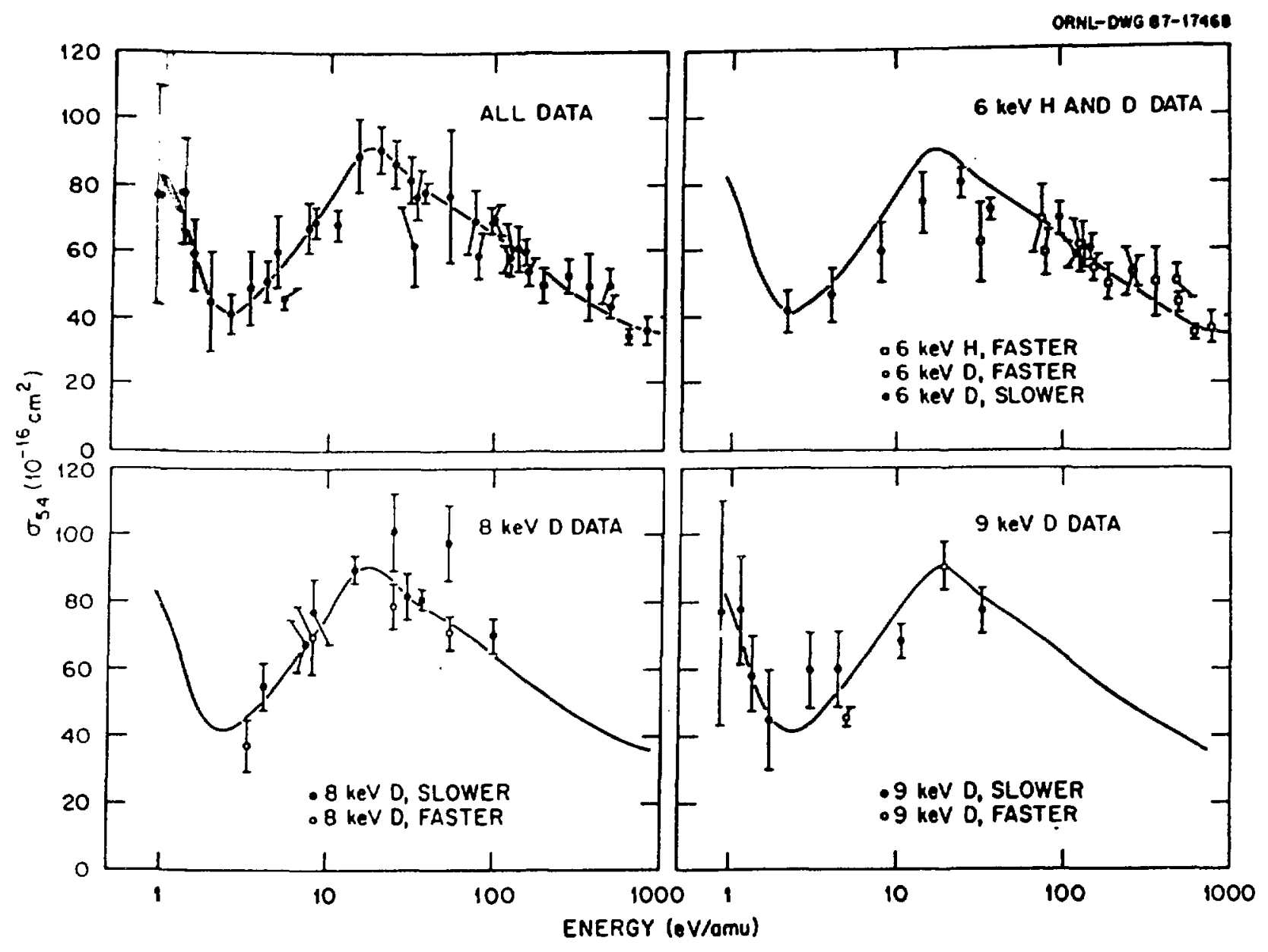

Fig. 6. Data for $05++H(D)$ collisions taken under different laboratory conditions. (a) All data, under all conditions, are plotted and a line is drawn to guide the eye through the scatter in the data points. The data taken w/th (b) $6 \mathrm{keV} \mathrm{H}$ and $D$, (c) $8 \mathrm{keV} \mathrm{D,} \mathrm{(d)} 9 \mathrm{keV} D$ beams are compared with the line from (a) representing the average of all the data.

For measurements below $2 \mathrm{eV} / \mathrm{amu}$ the sharp increase observed in $c_{54}$ is suggestive of the $1 / \mathrm{V}$ dependence predicted by the classical orbiting model. According to this model 191 , as the ion and reutral approach, a dipole is induced in the neutral causing an attractive force and possible orbiting trajectories. However, as pointed out by Henchman /17/, it may be inappropriate to arbitrarily appiy this simple classical orbicing plcture to electron-capture collisions, where the important curve crossings often occur at too large an internuciear separation for the incoming ion-neutral pair to sample the potential well caused by the ion-induced dipole.

Measurements $17 /$ for collisions of $\mathrm{N}^{5+}+\mathrm{H}(\mathrm{D})$ (see Fig. 5) show good agreement with theoretical calculations which indicate a rising cross section with decreasing $E_{\text {rel }}$. There is considerable scatter in the data that is believed to be primarlly due to the short term instabilities of the $\mathrm{N}^{5+}$ beam produced by the ion source. Comparing the $\mathrm{N}^{5+}$ measurements to the $0^{5+}$ measurements, one sees that even though the calculations predirt the same general trend with energy for both systems, the measured cross sections are quite different. 


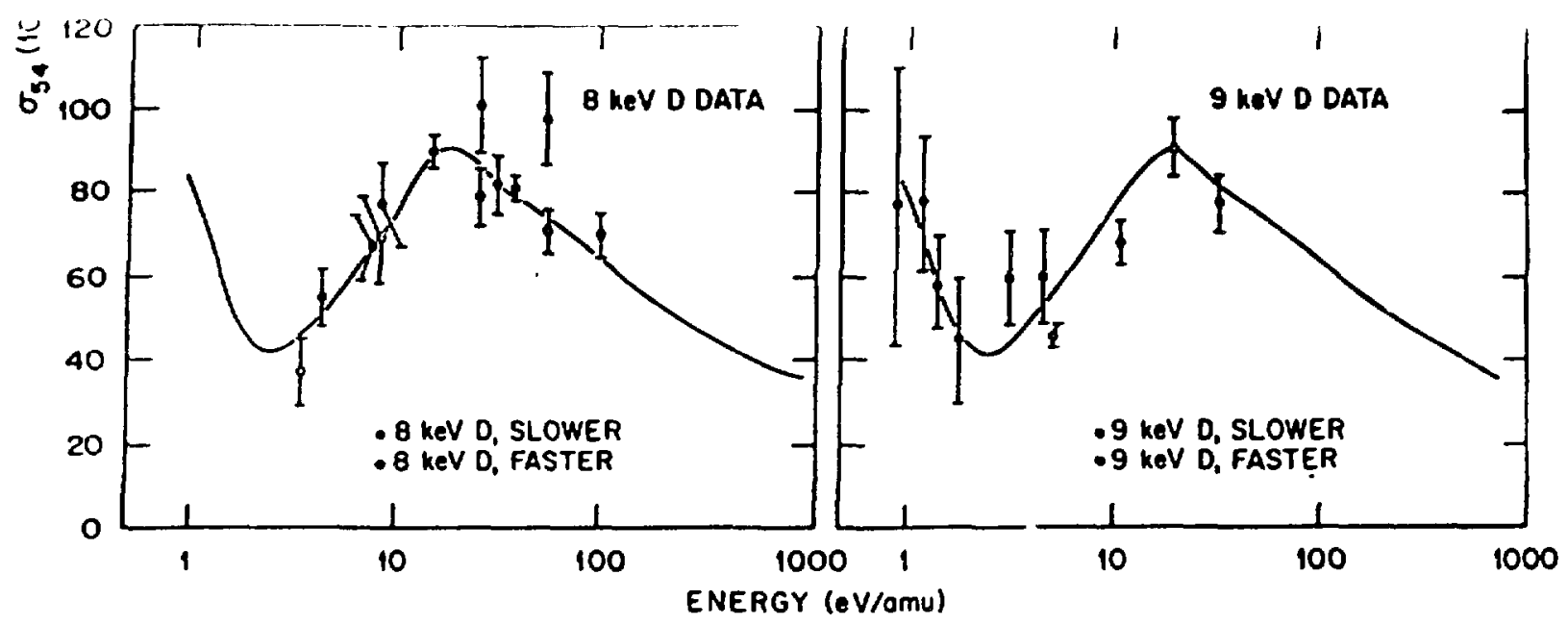

Fig. 6. Data for $05++H(D)$ collisions taken under different iaboratory conditions. (a) All data, under all conditions, are plotted and a line is drawn to gulde the eye through the scatter in the data points. The data taken with (b) $6 \mathrm{keV} \mathrm{H}$ and $\mathrm{D}$, (c) $8 \mathrm{keV} \mathrm{D}$, (d) $9 \mathrm{keV} D$ beams are compared with the line from (a) representing the average of all the data.

For measurements below $2 \mathrm{eV} / \mathrm{amu}$ the sharp increase observed in $\sigma_{54}$ is suggestive of the $1 / \mathrm{V}$ dependence predicted by the classical orbiting model. According to this model $/ 9 /$, as the ion and neutral approach, a dipole is induced in the neutral causing an attractive force and possible orbiting trajectories. However, as pointed out by Henchman /17/, it may be inappropriate to arbitrarily apply this simple classical orbiting picture to electron-capture collisions, where the important curve crossings often occur at too large an internuclear separation for the incoming lon-neutral pair to sample the potential well caused by the ion-induced dipole.

Measurements $17 /$ for collisions of $\mathrm{N}^{5+}+\mathrm{H}(\mathrm{D})$ (see Fig. 5) show/good agreement with theoretical calculations which indicate a rising cross section with decreasing $E_{\text {rel. }}$. There is considerable scatter in the data that is believed to be primarily due to the short term instabilities of the $\mathrm{N}^{5+}$ beam produced by the ion source. Comparing the $\mathrm{N}^{5+}$ measurements to the $0^{5+}$ measurements, one sees that even though the calculations predict the same general trend with energy for both systems, the measured cross sections are quite different.

The merged-beams technique was extended to other systems where additional comparison could be made. The measurements $/ 7 /$ for the $\mathrm{N}^{3+}+\mathrm{H}$ collisions are in excellent agreement with the theoretical calculations of Gargaud and Mccarroll /18/, Bienstock et al. $119 /$. Watson and Christensen 15/, and McCarroll and Valiron /20/. The $\mathrm{N}^{4+}$ measurements /7/ agree with the low energy calculations of feikert et al. /21/ where overlap exists ( $\approx \mathrm{eV} / \mathrm{amu})$. 
Having gained additional confidence in the merged-beams technique, we now re-examine the $0^{5+}$ results. A schematic representation of the diabatic potential energy curves for the $\mathrm{O}^{5+}+\mathrm{H}$ collision system is presented in Fig. 7, showing the approximate crossing distances of several product channels with the incident channel. Inspection of the energy dependence of Gargaud's $4 \mathrm{~s}$ ano $4 \mathrm{p}$ partial cross-section calculation $/ 16 /$ shows that the decrease observed in the expertimental data follows the calculated energy dependence for the $4 \mathrm{~s}$ cross section. The $4 p$ contribution to the calculated cross section, which is dominant at the lower energies, lacks the correct energy dependence to account for the experimental data.

In the next section the Landau-Zener model will be used to qualitatively investigate the dynamics of electron capture at low energies, i.e., to investigate the role of trajectory effects due to the ion-induced dipole on the electron capture cross section. Identification of final states is sought whose cross section is enhanced by the lon-induced dipole attraction, leading to an energy dependence similar to that observed.

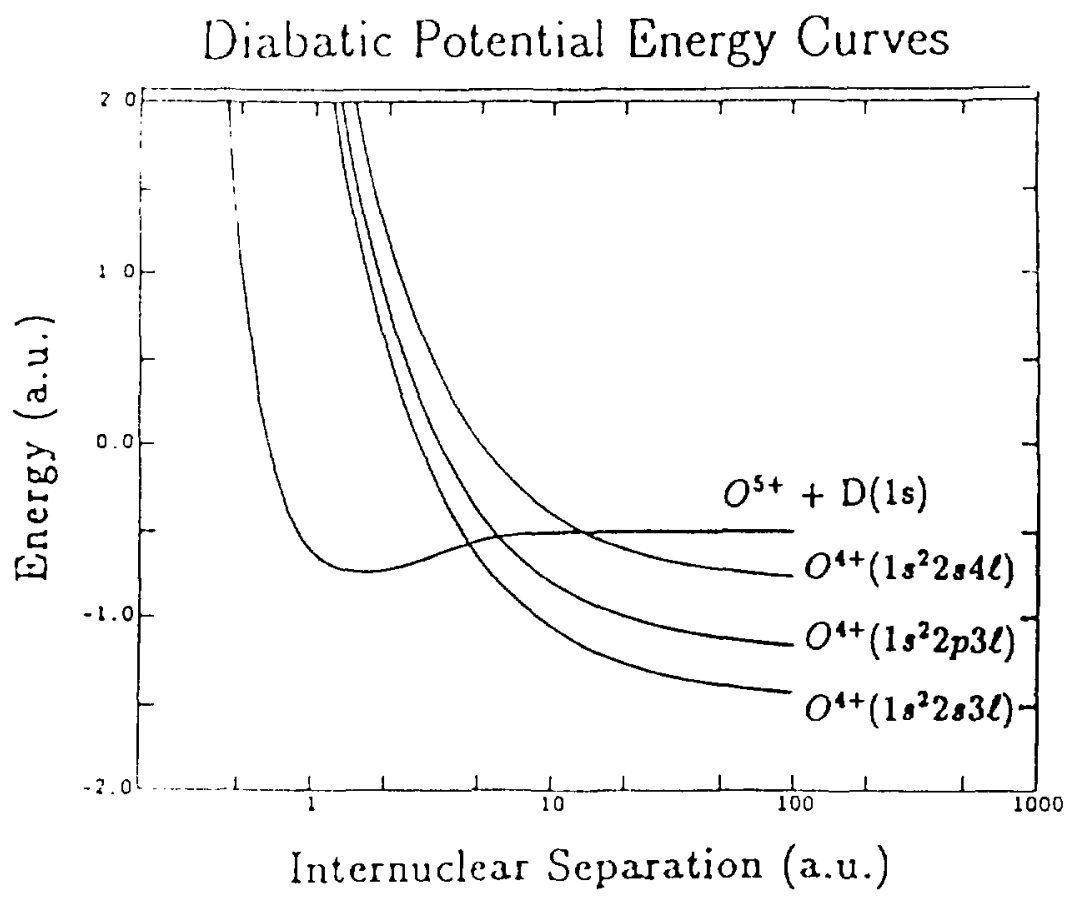

Fig. 7. Schematic representation of the diabatic potential curves for the $0^{5+}+D$ collision system showing the approximate crossing distances $\left(R_{c}\right)$ for several final states with the incident channel. The shallow well in the incldent channel is caused by the attractive ioninduced dipole potential.

4 - LANDAU-ZENER CALCULATIONS WITH CLASSICAL TRAJECTORIES

We have used the measured peak in the $0^{5+}$ cross section at 20 ev/amu to obtain estimates for the parameters necessary to perform Landau-Zener calculations. These calculations include trajectory effects due to the ion-induced-dipole attraction in the initial state.

The capture cross section is calculated /22/ by integrating the transition probability, $2 p(1-p)$, over the impact parameter $b$,

$$
0=2 \pi \int_{0}^{b_{\max }} 2 p(1-p) b d b
$$




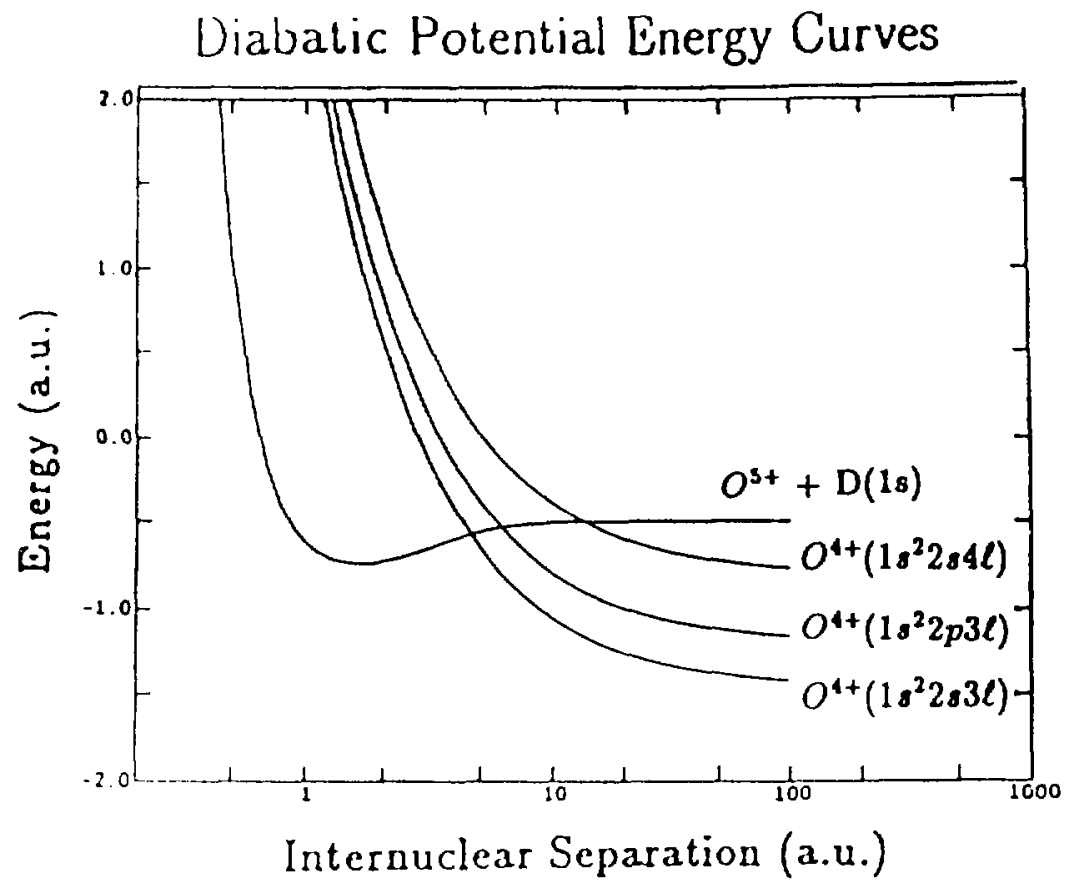

Fig. 7. Schematic representation of the diabatic potential curves for the $05++D$ colliston system showing the approximate crossing distances $\left(R_{c}\right)$ for several final states with the incident channel. The shallow well in the incident channel is caused by the attractive ioninduced dipole potential.

\section{4 - LANDAU-ZENER CALCULATIONS WITH CLASSICAL TRAJECTORIES}

We have used the measured peak in the $0^{5+}$ cross section at $20 \mathrm{eV} / \mathrm{amu}$ to obtain estimates for the parameters necessary to perform Landau-Zener calculations. These calculations include trajectory effects due to the ion-induced-dipole attraction in the initial state.

The capture cross section is calculated $/ 22 /$ by integrating the transition probability, $2 p(1-p)$, over the impact parameter $b$,

$$
\sigma=2 \pi \int_{0}^{b_{\max }} 2 p(1-p) b d b
$$

where $p$ is the Landau-zener probability and is given by

$$
p=\exp \left(-\frac{2 \pi \Delta^{2}}{v_{r} \mid H_{11^{\prime}-H_{22}}{ }^{\prime}}\right)
$$


$\Delta$ is the energy splitting at the avoided crossing, $R_{C} ; v_{r}$ is the radial component of the collision velocity; and $/ H_{11},-H_{22}, /$ is the difference in slopes of the diabatic potential energy curves at the crossing. Since the crossings occur at relatively large internuclear separations, the potential energy for the initial state can be approximated by that due to the ion-induced dipole, $-a_{4^{2}} / R^{4}$, ( $\alpha$ is the polarizability of $H$ ) and the final-state potential energy by $(q-1) / R$ (atomic units). $\Delta$ was estimated /23/ from the observed maximum in the cross section at $20 \mathrm{ev} / \mathrm{amu}$ and found to be $6.23 \times 10^{-3} \mathrm{a} . \mathrm{u}$. Equations (3)-(4) were evaluated with reactant trajectories which account for the ion-induced dipole. This resulted in straightforward modifications to $H_{11}, b_{\max }$, and $v_{r}$.

In order to perform calculations for different final states which cross the initial state at different internuclear separations, a new energy splitting was calculated using Butler and Dalgarno's $R_{c}{ }^{2} e^{-R_{c}}$ scaling $/ 24 /$ and by assuming, as is shown in the calculations of Gargaud $116 /$, that the peak in the cross section at $20 \mathrm{eV} / \mathrm{amu}$ is due to capture to the $1 \mathrm{~s}^{2} 2 \mathrm{~s} 4 \mathrm{~s}$ state of $0^{4+}$ which has a crossing at $\approx 10 \mathrm{a} . u$. By scaling the energy splitting to the inter.luclear separation appropriate for the $1 s^{2} 2 s 4 p$ final state $(\approx 12$ a.u.), the Landau-Zener cross section for capture to the $4 p$ was calculated and found to be consistent to the results of Gargaud and McCarroll /16/. By comparing Landau-zener calculations with and without the potential due to ion-induced dipole, the effect due to considering realistic or straight-line trajectories could be estimated. It was found that for capture to the $254 \mathrm{~s}$ and $2 \mathrm{~s} 4 \mathrm{p} s \mathrm{states}$ there was negligible cross-section enhancement due to the attractive potentlal at $1 \mathrm{eV} / \mathrm{amu}$.

Final-state cross sections that are enhanced by trajectory effects and, thereby, more likely to produce the correct energy dependence at low energies will occur at lower $R_{C}$ than that populating 2s4s, where the ion-induced dipole attraction is stronger (see Fig. 7 ). Crossings populating $n=3$ product states occur at internuclear separations of $\approx 4$ a.u. and. therefore, $\Delta$ appropriately scaled increases by a factor of $\approx 83$ relative to that found for the $4 \mathrm{~s}$ crossing. This causes the calculated cross section for this state to be shifted to higher energies, leaving negligible contribution around $1 \mathrm{eV} / \mathrm{amu}$.

We next consider states such as $1 s^{2} 2 p 3 s$ which involve capture into the $3 s$ state plus a coreexcitation $(2 s-2 p)$, the effect of the excitation being a lowering of $\Delta$ by the factor $S\left(R_{c}\right)=$ $0.5 e^{-0.4 R_{C}} \mathrm{124/}$. In an independent-electron model $\Delta$ can be estimated by a product of $S(R C)$ and the above $R$ scaling for single capture $124 /$. The result is that $H_{12}$ is increased by a factor of 1.6 relative to $\Delta$ for capture to $1 \mathrm{~s}^{2} 2 \mathrm{~s} 4 \mathrm{~s}$. The calculated cross sections for this channel with both reaistic and straight-line trajectories are shown in Fig. 8 . Also shown in the flgure are the Landau-Zener calculations for single capture to 2s4s in which trajectory effects were calculated to be negligible. The cross section for transfer plus excitation is $\approx 10 \%$ of that due to $4 \mathrm{~s}$ at around $20 \mathrm{eV} / \mathrm{amu}$. It is shown that for lower energies the calculation for the $2 \mathrm{p} 3 \mathrm{~s}$ final state exhibits the measured energy dependence when realistic trajectories are used.

Of course, finding a final state with the correct energy dependence does not mean that it contributes significantly to the total cross scction. At 20 ev/amu the dominant final states $(4 \ell)$ cross the initial state at $r=\approx 10-14 \mathrm{a} . u$ and thereby reduce the initial flux reachlng the inner channels. For lower energies the "leaction window" tends to move toward larger internuclear separations making these crossing more adiabatic, and thereby even further blocking the initial flux from reaching the inner crossings. To estimate the transition probablitity to the $1 \mathrm{~s}^{2} 2 \mathrm{p} 3 \mathrm{~s}$ final state in the presence of the $4 \ell$ final states crossing the initial channel at the larger $R_{c}{ }^{s}$, a Multichannel-Landau-Zener treatment $(M C l z)(25 /$ 

Gargaud and Mccarroll $116 \%$. By comparing Larsou-Zener siculations with and without the potential due to ion-induced dipole, the effect sue to considering realistic or straight-iine trajectories could be estimated. It was found that for cyture to the $2 \mathrm{~s} 45$ and $254 \mathrm{p}$ states there was negligible cross-section enhancement due to the stractive potential at 1 ev/amu.

Fina -state cross sections that are enhanced by trajectory sffects and, thereby, more likely to produce the correct energy dependence at low energies 111 occur at lower $R_{c}$ than that populating 2s4s, where the ion-induced dipole attraction is stronger (see Fig. 7). Crossings populating $n=3$ product states occur at internuclear separations of $\approx 4$ a.u. and, therefore, $\Delta$ appropriateiy scaled increases by . factor of $\approx 83$ relative to that found for the $4 \mathrm{~s}$ crossing. This causes the calculated cross section for this state to be shifted to higher energies, leaving negligible contribution oround $1 \mathrm{eV} / \mathrm{amu}$.

We next consider states such as $1 s^{2} 2 \mathrm{p} 3 \mathrm{~s}$ which involve capture into the 35 state plus a coreexcitation $(2 \mathrm{~s}-2 \mathrm{D})$. the effect of the excitation teing a lowering of $\Delta$ by the factor $S\left(R_{C}\right)=$ $0.5 e^{-0.4 R_{C}} / 24 \%$. In an independent-electron model a can be estimated by a product of $S(R C)$ and the above $R$ scaling for single capture $/ 24 \%$. The result is that $H_{12}$ is increased by a factor of 1.6 relative to $\Delta$ for capture to $15^{2} 251 \mathrm{~s}$. The calculated cross sections for this channel with both realistic and stralght-line trajectories are shown in Fig. 8 . Also shown in the figure are the Landau-Zener calculations for :ingle capture to 2545 in which trajectory effects were calculated to be negligible. The cross section for transfer plus excitation is $\approx 10 \%$ of that due to $4 \mathrm{~s}$ at around $20 \mathrm{ev} / \mathrm{amu}$. It is shown that for lower energies the calculation for the 2035 final state exhibits tho measured energy dependence when realistic
trajectories are used.

of course, finding a final state with the correce energy dependence does not mean that it contributes significantly to the total cross section. At $20 \mathrm{eV} /$ amu the dominant final states (48) cross the initial state at $r==10-14$ a.u. and thereby reduce the initial flux reaching the inner channels. For lower energies the "reaction window" tends to move toward larger internuclear separations making these crossing more adiabatic, and thereby even further blocking the initial flux from reaching the inner crossings. To estimate the transition probability to the $1 s^{2} 2 \mathrm{p} 3 \mathrm{~s}$ final state in the prance of the 4 e final states crossing the initial channel at the larger $R_{C}{ }^{\prime} s$, a Multichannel landau-zi'.er treatment (MCLZ) $/ 25 /$ is used resulting in the totel $2 p 3 s$ transition probability

$$
p_{2 \rho 3 s}=2 p_{1} p_{2} p_{3} p_{4}\left(1-p_{4}\right)
$$


tion for capture to the $4 p$ was calculated and found to be Gargaud and McCarroll /16/. By comparing Landau-Zener calcusststent to the results of potential due to ion-induced dipole, the effect due to considering mis with and without the trajectories could be estimated. It was found that for capture to ine $2 \mathrm{~s} 4 \mathrm{~s}$ and $2 \mathrm{~s} 4 \mathrm{p}$ states there was negligible cross-section enhancement due to the attractivo potential at 1 ey/amu.

Final-state cross sections that are enhanced by trajectory effects \&ad, thereby, more likely
to produce the correct energy dependence at low energies will coctr to produce the correct energy dependence at low energies will cccir at lower $R_{c}$ than that
populating $254 \mathrm{~s}$, where the ion-induced dipole attraction is Crossings populating $n=3$ product states occur at internuclear separations (see Fig. 7 ). therefore, $\Delta$ appropriately scaled increases by a factor of $=53$ relative to that found for the $4 \mathrm{~s}$ crossing. This causes the calculated cross section for this state to be shifted to higher energies, leaving negligible contribution around 1 ey/amu.

We next consider states such as $1 \mathrm{~s}^{2} 2 \mathrm{p} 3 \mathrm{~s}$ which involve capture ints is $3 \mathrm{~s}$ state plus a coreexcitation $(2 s-2 p)$, the effect of the excitation being a lowering of $I$ by the factor $S\left(R_{c}\right)=$ $0.5 e^{-0.4 R c} / 24 /$. In an independent-electron model $\Delta$ can be estimated by a product of $S(R C)$ and the above $R$ scaling for single capture $124 /$. The result is that $H_{12}$ is increased by a factor of 1.6 relative to $\Delta$ for capture to $1 \mathrm{~s}^{2} 2 \mathrm{~s} 4 \mathrm{~s}$. The calculated cross sections for this channel with both realistic and straight-line trajectories are shown in Fig. 8 . Also shown in the figure are the Landau-zener calculations for single capture to $2 \mathrm{~s} 4 \mathrm{~s}$ in which trajectory effects were calculated to be negligible. The cross section for trarsfrar plus excltation $1 \mathrm{~s} \approx 10 \%$ of that due to $4 \mathrm{~s}$ at around $20 \mathrm{eV} / \mathrm{amu}$. It is shown that for lower energies the calculation for the $2 p 3 s$ final state exhibits the measured energy dependence when realistic
trajectories are used.

Of course, finding a final state with the correct energy dependence does not mean that it contributes significantly to the total cross section. At $20 \mathrm{eV} / \mathrm{amu}$ the dominant final states (4l) cross the injtial state at $r=\approx 10-14 \mathrm{a} . u$. and thereby reduce the inftial flux reaching the inner channels. For lower energies the "reaction window" tends to move toward larger internuclear separations making these crossing more adiabatic, and thereby even further probability to the $1 s^{2} 2 p 3 s$ final state in the presence of the of initial channel at the larger $R_{c}{ }^{\prime} s$, a Multichannel-Landau-Zener lreatill states crossing the resuiting in the total $2 p 35$ transition probability

$$
p_{2 p 3 s}=2 p_{1} p_{2} p_{3} p_{4}\left(1-p_{4}\right)
$$




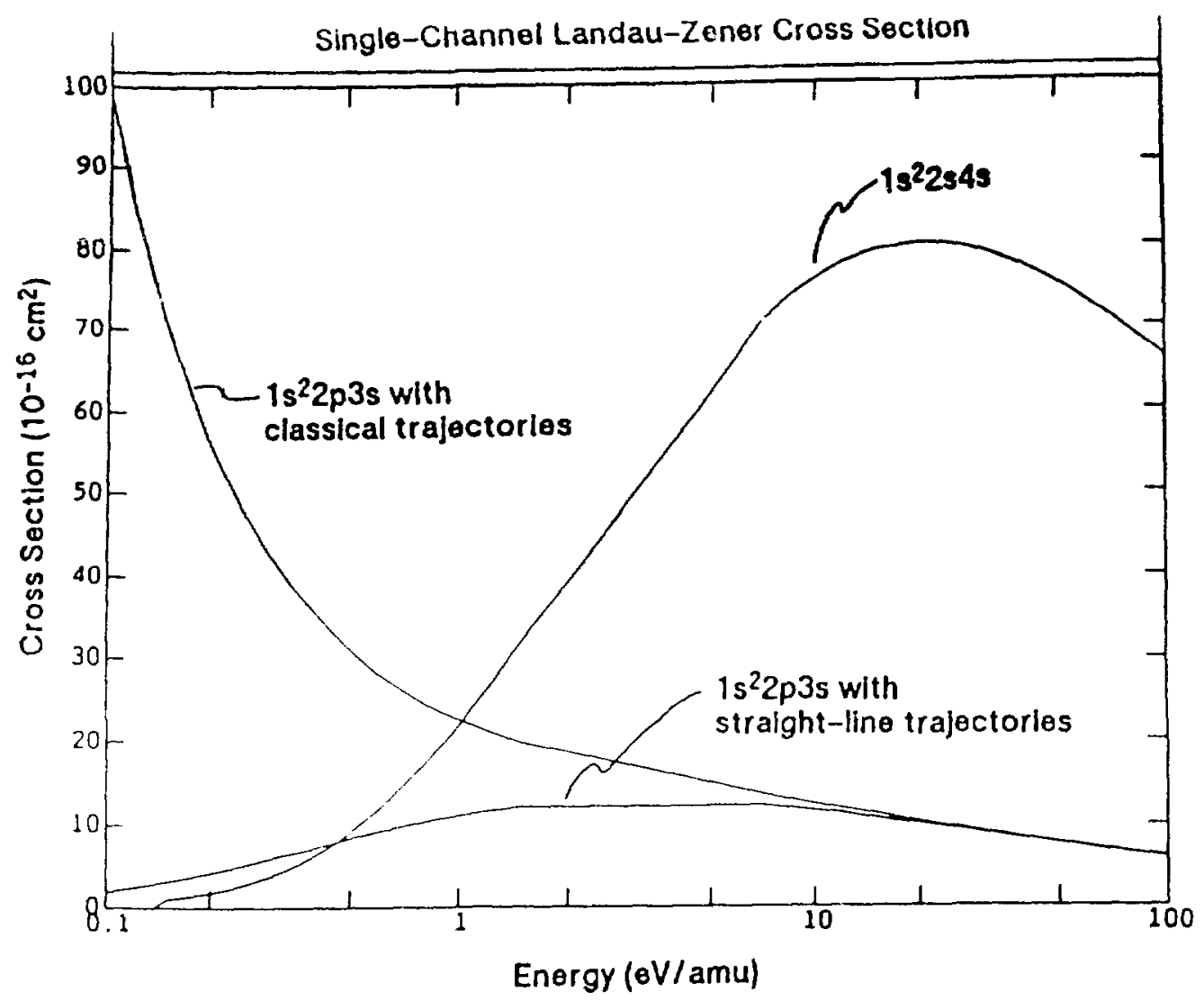

Fig. 8. Single-channel Landau-zener calculations for capture into the $1 s^{2} 2545$ and $1 s^{2} 2 p 3 s$ states of $0^{4+}$. Classical trajectories were used which fincluded effects due to the foninduced dipole potential. For capture to the $1 s^{2} 2 p 3 s$ state, the Landau-Zener calculations with straight line trajectories are also shown.

where $p_{1}, \ldots p_{4}$ are the Landau-Zener probabilities [Eq. (4)] for the participating $4 s, 4 p, 4 d$, and $2 p 3 s$ product states, respectively. Integration of this transition probability over impact parameters [see Eq. (3)] leads to an almost negligible contribution to the total cross section below $1 \mathrm{ev} / \mathrm{amu}$. However, our treatment here is only an approximation to a more appropriate coupled molecular state calculation, where detalls of the fonic core are inciuded.

$5-\underline{\text { SUMMARY }}$

The merged-beam appiratus has been used to measure absolute total electron capture for several multicharged ions colliding with ground state $H$ over three decades of energy, from less than $1 \mathrm{eV} / \mathrm{amu}$ to over $1000 \mathrm{eV} / \mathrm{amu}$. These systems provide benchmarks to compare with previous measurements and theory where overlap exists. A discrepancy between theory and measurements exists for the $\mathrm{O}^{5+}+\mathrm{H}(\mathrm{D})$ at low energies. To identify possible final states which exhibit the observed energy dependence, a Landau-Zener calculation was performed whlch incorporates the ion-induced dipole attraction between the reactants. It was found that the final state $1 \mathrm{~s}^{2} 2 \mathrm{p} 3 \mathrm{~s}$ of $\mathrm{0}^{4+}$, which includes capture plus excitation, exhibits the observed energy dependence if competing channels are ignored, and that this channel is strongly influenced by the attractive potential. However, to assess the relative contribution of this channel to the total cross section, comprehensive coupled-state calculations will be required. 
This research was sponsored by the Division of Chemical Sciences, U.S. Department of Energy, under Contract No. DE-AC05-840R21400 with Martin Marietta Energy Systems, Inc. This research would not have been possible without the stable, high-intensity beams of multiply charged ions produced by the ORNL-ECR ion source. We are indebted to C. Bottcher and R. K. Janev for many fruitful discussions. Special thanks are due to M. Gargaud, R. McCarroll, C. Bottcher, and $T$. G. Heil for comunicating their theoretical results prior to publication.

M. S. Huq was appointed through a Postgraduate Research Training Program, administered by Oak Ridge Associated Universities (ORAU) for the U.S. Department of Energy.

\section{7 - REFERENCES}

11 W. Fritsch and C. D. Lin, J. Phys. B: At. Mol. Phys. 17, 3271 (1984).

121 T. A. Green, E. J. Shipsey, J. C. Browne, Phys. Rev. $\bar{A} 25,1364$ (1982).

$13 /$ C. Bottcher and T. G. Heil, Chem. Phys. Lett. 86, 506 (1982).

14/ M. Gargaud and R. McCarroll, J. Phys. B: At. Mol. Opt. Phys. 21, 513 (1988).

15/ W. D. Watson and R. B. Christensen, Astrophys. J. 231, 627 (1979).

161 C. C. Havener, M. S. Huq, H. F. Krause, P. A. Schulz, and R. A. Phaneuf, submitted to Physical Review A.

$17 /$ M. S. Huq, C. C. Havener, and R. A. Phaneuf (prepared for Physical Review A).

181 R. E. Olson, in Electronic and Atomic Collisions, N. Oda and K. Takayanagt, eds., 1980, North-Holland Pub. Co., pp. 391-405.

19/ G. Gioumousis and D. P. Stevenson, J. Chem. Phys. 29, 294 (1958).

$110 /$ R. A. Phaneuf, I. Alvarez, F. W. Meyer, and D. H. Crandall, Phys. Rev. A 26, 1892 (1982).

111/ M. Gargaud and R. McCarroll, J. Phys. B: At. Mol. Opt. Phys. 21, 513 (1988).

$112 /$ 0. G. Larsen and K. Taulbjerg, J. Phys. B: At. Mol. Phys. $\frac{17}{45} 23$ (1984).

$113 /$ D. Dijkkamp, Yu. S. Gordeev, A. Brazuk, A. G. Orentje, F. J. de Heer, J. Phys. B: At. Mol. Phys. 18,737 (1985).

/14/ R. E. Olson and M. Kimura, J. Phys, B: At. Mol. Phys. 15, 4231 (1982).

$115 /$ F. W. Meyer, A. M. Howald, C. C. Havener, and R. A. Phaneuf Phys. Rev. A 32,3310 (1985).

$116 /$ M. Gargaud, Thesis, L'Université de Bordeaux I, 1987; M. Gargaud and R. McCarroll, in preparation.

171 M. Henchman, in Ion-Molecular Reactions, J. L. Franklin, ed., Vol. I, Plenum Publishing Co. (1972), pp. 101-259.

$118 /$ M. Gargaud and R. McCarroll, J. Phys. B 18, 463 (1985).

$119 /$ S. Bienstock, A. Dalgarno, and T. G. HelT, Phys. Rev. A 29, 503 (1984).

$120 /$ R. McCarrall and P. Valiron, Astron. Astrophys. 78,177 (1979).

$121 /$ C. A. Feickert, R. J. Blint, G. T. Suratt, and W. D. Watson, Astrophys. J. 286, 371 (1984).

1221 e.g., F. W. Meyer, A. M. Howald, C. C. Havener, and R. A. Phaneuf, Phys. Rev. Lett. 54 , $2663(1985)$.

/23/ R. E. Olson, F. T. Smith, and E. Bauer, Appl. Opt. 10, 1848 (1971).

$124 /$ S. E. Butler and A. Dalgarno, Astrophys. J. 241,838 (1980).

1251 A. Salop and R. E. 01 son, Phys. Rev. A 13,1312 (1976). 